\title{
DANIEL ÁNGEL FRASSINETTI CABEZAS, 1939-2010: obituary, bibliography and a list of his taxa*
}

\author{
Sven N. Nielsen ${ }^{1}$ and Jhoann Canto ${ }^{2}$
}

\begin{abstract}
Institut für Geowissenschaften, Christian-Albrechts-Universität zu Kiel, Ludewig-Meyn-Str. 10, 24118 Kiel, Germany. e-mail. nielsen@gpi.uni-kiel.de

2 Museo Nacional de Historia Natural, Área Vertebrados, casilla 787, Santiago, Chile.e-mail: jhoanncanto@gmail.com
\end{abstract}

\section{Obituary}

Daniel Frassinetti was born on July 26, 1939 in Iquique and died on April 9, 2010 in Santiago, Chile. He obtained the title of Teacher of General Basic Education in 1959 from Escuela Normal José Abelardo Núñez and worked as a teacher for several years. He came to Santiago in 1971, where he joined the Museo Nacional de Historia Natural as a technician. Quickly he became research assistant and in 1975 he ascended to researcher of the Geology Section. In 1991 he became head of the Paleontology Section and was editor of the Boletín del Museo Nacional de Historia Natural and the museum's Publicación Ocasional for more than 30 years. In 1995 he obtained a degree in Education from the Universidad Metropolitana de Ciencias de la Educación. Daniel Frassinetti retired in the year 2008, but continued his investigations as Emeritus Curator at the museum. He was always generous with information about paleontology, but rarely talked about his private life. For some years, he fought silently and bravely against a cancer without many people knowing about it. A fight he eventually lost.

'Don Daniel', as he was known to many of his students, colleagues and friends, worked mostly on Neogene mollusks and Quaternary mammals from Chile. The investigation of mollusks was done closely together with his friend Vladimir Covacevich (SERNAGEOMIN) with whom he also assembled the largest available collection of Neogene marine invertebrates from Chile and published many papers on the subject. After Vladimir Covacevich's untimely death in 1997, Daniel Frassinetti continued to publish, but he was missing the collaboration of a counterpart. The appearance of one of the authors (SNN), beginning a Ph.D. thesis in the year 2000 right at the core of his research focus, did not lead Daniel to hinder that work, but to open his arms wide

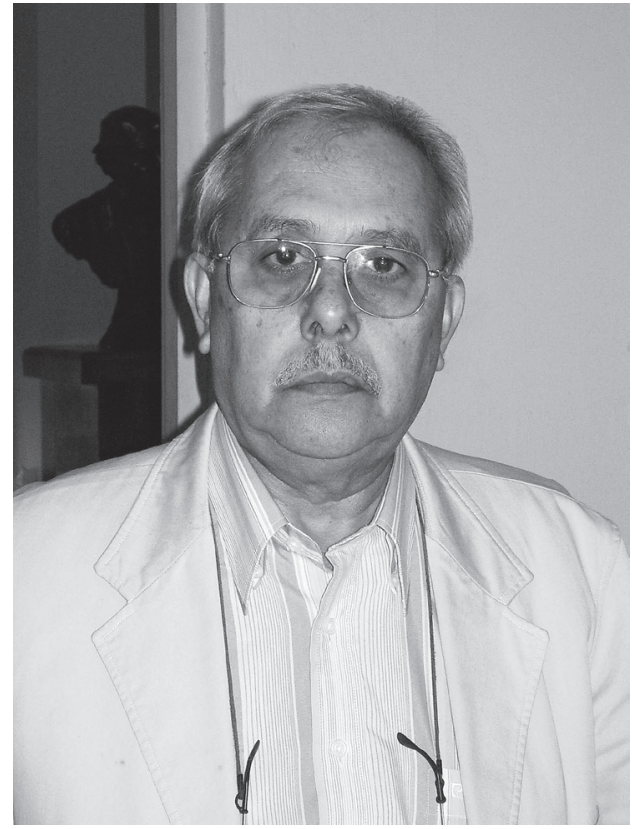

for a new fruitful collaboration. Daniel Frassinetti was the first palaeontologist of the museum to collaborate closely with colleagues from the US, Europe and other countries and helped national and international students to develop their investigations without reservations or restrictions. With this, he made Chilean paleontology much more visible on a global scale.

To our knowledge, four species were named after Daniel Frassinetti, the Miocene gastropods Chorus frassinettii DeVries, 1997 and Zonaria (Zonaria) frassinettii Groves \& Nielsen, 2003, the Pliocene crustacean Trichopeltarion frassinetti Feldmann, Schweitzer \& Encinas, 2010, and the extant lizard Liolaemus frassinetti Núñez, 2007.

We remember Daniel as a very kind man, very helpful and encouraging. He will long be remembered by those who knew him and even longer through his scientific contributions.

A preliminary version was published in the abstract volume of the II Simposio - Paleontología en Chile (Concepción, 2010), which was dedicated to the memory of Daniel Frassinetti. 


\section{Bibliography}

Bahamonde, N.; Frassinetti, D. 1980. Lebucarcinus n. gen. del Mioceno de Chile (Crustacea, Decapoda, Brachyura). Boletín del Museo Nacional de Historia Natural 37: 275-279. Santiago.

Canto, J.; Frassinetti, D. 2008. La biodiversidad extinta en Chile. In Biodiversidad de Chile. Patrimonio y Desafíos, Second Edition (Comisión Nacional del Medio Ambiente, CONAMA). Ocho Libros Ediciones: 64-70. Santiago.

Canto J.; Yáñez, J.; Frassinetti, D. 2009. Mamíferos Fósiles de Chile. In Mamíferos de Chile, Second Edition (Muñoz, A.; Yáñez, J.; editors). Ediciones CEA: 285-297. Santiago.

Canto J.; Fariña, R.; Yáñez, J.; Frassinetti, D. 2009. Paleoecología. In Mamíferos de Chile, Second Edition (Muñoz, A.; Yáñez, J.; editors). Ediciones CEA: 299-304. Santiago.

Charrier, R.; Wyss, A.R.; Norell, M.A.; Flynn, J.J.; Novacek, M.J.; McKenna, M.C.; Swisher III, C.C.; Frassinetti, D.; Salinas, P. 1990. Hallazgo de mamíferos fósiles del Terciario Inferior en el sector de Termas del Flaco, Cordillera Principal, Chile Central: Implicaciones paleontológicas, estratigráficas y tectónicas. Segundo Simposio sobre el Terciario de Chile, Actas: 73-84. Concepción.

Covacevich, V.; Frassinetti, D. 1977. El género Encope en el Plioceno del norte de Chile (Echinodermata, Echinoidea). Boletín del Museo Nacional de Historia Natural 35: 49-60. Santiago.

Covacevich, V.; Frassinetti, D. 1980. El género Ficus en el Mioceno de Chile Central con descripción de F. gayana sp. nov. Gastropoda: Ficidae. Boletín del Museo Nacional de Historia Natural 37: 281-294. Santiago.

Covacevich, V.; Frassinetti, D. 1983. Diconoficus, nuevo subgénero de Ficus (Mollusca: Gastropoda) en la Formación Navidad, Mioceno, Chile Central. Revista Geológica de Chile 19-20: 105-110.

Covacevich, V.; Frassinetti, D. 1986. El género Cancellaria en el Mioceno de Chile con descripción de cuatro especies nuevas (Gastropoda: Cancellariidae). Revista Geológica de Chile 28-29: 33-67.

Covacevich, V.; Frassinetti, D. 1990. La fauna de Lo Abarca: hito biocronoestratigráfico y paleoclimático en el Terciario Superior marino de Chile Central. Segundo Simposio sobre el Terciario de Chile, Actas: 51-71.

Covacevich, V.; Frassinetti, D. 1993. Principales cambios en los moluscos marinos del Terciario Superior de Chile central $\left(33^{\circ}-34^{\circ}\right.$ Lat.S). Amici Molluscarum, Sociedad Chilena de Malacología, Boletín 2: 11-12.
Covacevich, V.; Frassinetti, D.; Alfaro, G. 1992. Paleontología y condiciones de depositación del Mioceno marino en las nacientes del Río Futa, Valdivia, Chile. Boletín del Museo Nacional de Historia Natural 43: 143-154. Santiago.

DeVries, T.J.; Frassinetti, D. 2003. Range extensions and biogeographic implications of Chilean Neogene mollusks. Boletín del Museo Nacional de Historia Natural 52: 119-135. Santiago.

Flynn, J.J.; Novacek, M.J.; Dodson, H.E.; Frassinetti, D.; McKenna, C.; Norell, M.A.; Sears, K.E.; Swisher III, C.C.; Wyss, A.R. 2002. A new fossil mammal assemblage from the southern Chilean Andes: implications for geology, geochronology, and tectonics. Journal of South American Earth Sciences 15: 285-302.

Frassinetti, D. 1974. El género Venus en la colección de fósiles Terciarios y Cuartarios de R.A. Philippi (1887). Boletín del Museo Nacional de Historia Natural 33: 43-51. Santiago.

Frassinetti, D. 1975. El género Artemis en la Colección de fósiles terciarios y cuaternarios de R. A. Philippi (1887). Boletín del Museo Nacional de Historia Natural 34: 217-225. Santiago.

Frassinetti, D. 1978a. Publicaciones. II: Museos e Investigación; Primeras Jornadas Museológicas Chilenas. Revista Muchi 7: 55-57.

Frassinetti, D. 1978b. Conservación de materiales paleontológicos. IV: Organización de Museos; Primeras Jornadas Museológicas Chilenas, Revista Muchi 7: 110-111.

Frassinetti, D. 1978c. Matanziella, nuevo subgénero de Bivalvia (Mollusca: Lucinidae) en el Mioceno de Chile Central. Revista Geológica de Chile 5: 49-54.

Frassinetti, D. 1980. Sección Geología. In G. Mostny (Ed.) Biobibliografía Museo Nacional de Historia Natural 1830-1980: 395 p. Santiago.

Frassinetti, D. 1982. Bibliografía escogida y comentada sobre mamíferos fósiles de Chile. Publicación Ocasional Museo Nacional de Historia Natural 37: 24 p. Santiago.

Frassinetti, D. 1982. Consideraciones sobre el estado actual de las investigaciones sobre mamíferos fósiles chilenos. Actas Primer Encuentro de Mastozoólogos Chilenos, Talca. Publicación Ocasional Museo Nacional de Historia Natural 38: 57-59. Santiago.

Frassinetti, D. 1985. Restos de mastodontes en Alto de Boroa y antecedentes de otros hallazgos en la Región de La Araucanía. Boletín Museo Regional de La Araucanía 2: 91-96.

Frassinetti, D. 1997. Moluscos del Plioceno Superior marino de la Isla Guafo, Sur de Chile. Parte I. Bivalvia. Boletín del Museo Nacional de Historia Natural 46: 55-79. Santiago. 
Frassinetti, D. 2000. Moluscos del Plioceno Superior marino de la Isla Guafo, sur de Chile. Parte II. Gastropoda. Boletín del Museo Nacional de Historia Natural 49: 131-161. Santiago.

Frassinetti, D. 2001. Moluscos bivalvos y gastrópodos del Mioceno marino de Isla Stokes, Sur de Chile. Boletín del Museo Nacional de Historia Natural 50: 73-90. Santiago.

Frassinetti, D. 2004. Moluscos fósiles del Mioceno marino de Isla Ipún, sur de Chile. Boletín del Museo Nacional de Historia Natural 53: 71-83. Santiago.

Frassinetti, D. 2006. Moluscos fósiles del Mioceno marino de islas Crosslet y Hereford (Golfo Tres Montes, Aisén, Chile). Boletín del Museo Nacional de Historia Natural 55: 61-74. Santiago.

Frassinetti, D.; Alberdi, M.T. 2000a. Revision y estudio de los restos fósiles de mastodontes de Chile (Gomphotheriidae): Cuvieronius hyodon, Pleistoceno Superior. Estudios Geológicos 56: 197-208.

Frassinetti, D.; Alberdi, M.T. 2000b. Presencia de Hippidion y Equus (Amerhippus) (Mammalia, Perissodactyla) y su distribución en el Pleistoceno Superior de Chile. Estudios Geológicos 56: 279-290.

Frassinetti, D.; Alberdi, M.T. 2001. Los macromamíferos continentales del Pleistoceno Superior de Chile: Reseña historica, localidades, restos fósiles, especies y dataciones conocidas. Estudios Geológicos 57: 53-69.

Frassinetti, D.; Alberdi, M.T. 2005. Presencia del género Stegomastodon entre los restos fósiles de mastodontes de Chile (Gomphotheriidae), Pleistoceno superior. Estudios Geológicos 61: 101-107.

Frassinetti, D.; Azcárate, V. 1974. Presencia de Megatherium en los alrededores de Santiago (Chile). Boletín del Museo Nacional de Historia Natural 33: 35-42. Santiago.

Frassinetti, D.; Covacevich, V. 1981a. Architectonicidae en la Formación Navidad, Mioceno, Chile Central, Parte I. Helicinae (Mollusca: Gastropoda). Revista Geológica de Chile 13-14: 35-47.

Frassinetti, D.; Covacevich, V. 1981b. Architectonicidae en la Formación Navidad, Mioceno, Chile Central. Parte II. Architectonica (Architectonica) nobilis karsteni Rutsch, 1934 (Mollusca: Gastropoda). Boletín del Museo Nacional de Historia Natural 38: 147-154. Santiago.

Frassinetti, D.; Covacevich, V. 1982. Architectonicidae en la Formación Navidad, Mioceno, Chile Central. Parte III. Architectonicinae. Boletín del Museo Nacional de Historia Natural 39: 101-109. Santiago.

Frassinetti, D.; Covacevich, V. 1984. Estudio del género Glycymeris da Costa, 1778 en el Terziario de Chile con descripción de dos nuevas especies (Mollusca: Bivalvia). Boletín del Museo Nacional de Historia Natural 40: 107-133. Santiago.

Frassinetti, D.; Covacevich, V. 1991. Nueva especie de Glycymerididae (Molusca, Bivalvia) para el Mioceno del Golfo Tres Montes (Aisén, Chile). Boletín del Museo Nacional de Historia Natural 42: 121-129. Santiago.

Frassinetti, D.; Covacevich, V. 1993. Bivalvos del Mioceno marino de Matanzas (Formación Navidad, Chile Central). Boletín del Museo Nacional de Historia Natural 44: 73-97. Santiago.

Frassinetti, D.; Covacevich, V. 1995. Moluscos del Plioceno Superior marino de Isla Guamblín, Archipélago de los Chonos, sur de Chile. Revista Geológica de Chile 22: 47-73.

Frassinetti, D.; Covacevich, V. 1999. Invertebrados fósiles marinos de la Formación Guadal (Oligoceno Superior-Mioceno Inferior) en Pampa Castillo, Región de Aisén, Chile. Servicio Nacional de Geología y Minería, Boletín 51: 96 p.

Frassinetti, D.; Salinas, P. 1986. Nuevos hallazgos de mastodontes ocurridos en Chile. Noticiario Mensual Museo Nacional de Historia Natural 311: 3-6. Santiago.

Le Roux, J.P.; Gómez, C.; Venegas, C.; Fenner, J.; Middleton, H.; Marchant, M.; Buchbinder, B.; Frassinetti, D.; Marquardt, C.; Gregory-Wodzicki, K.M.; Lavenu, A. 2005. Neogene-Quaternary coastal and offshore sedimentation in north central Chile: Record of sealevel changes and implications for Andean tectonism. Journal of South American Earth Sciences 19: 83-98.

Nielsen, S.N.; Frassinetti, D. 2003. New and little known species of Pseudolividae (Gastropoda) from the Tertiary of Chile. The Nautilus 117: 91-96.

Nielsen, S.N.; Frassinetti, D. 2007a. The Neogene Volutidae (Gastropoda, Neogastropoda) from the Pacific coast of Chile. Journal of Paleontology 81: 82-102.

Nielsen, S.N.; Frassinetti, D. 2007b. The Miocene Architectonicidae (Gastropoda) of Chile. Paläontologische Zeitschrift 81: 291-303.

Nielsen, S.N.; Frassinetti, D. 2008. Ipunina, a new genus perhaps of Litiopidae (lower Caenogastropoda) from the Neogene of southern Chile. Journal of Molluscan Studies 74: 253-257.

Nielsen, S.N.; Frassinetti, D.; Bandel, K. 2004. Miocene Vetigastropoda and Neritimorpha (Mollusca, Gastropoda) of Central Chile. Journal of South American Earth Sciences 17: 73-88.

Núñez, H.; Staford, T.W. Jr.; Frassinetti, D. 2005. Primer registro de fósiles de Liolaemus en Chile (Reptilia, Sauria). Noticiario Mensual Museo Nacional de Historia Natural 356: 3-7. Santiago. 
Osorio, C.; Frassinetti, D.; Bustos, E. 1983. Taxonomía y Morfometría de Venus antiqua antiqua King y Broderip, 1835 (Mollusca, Bivalvia, Veneridae). Revista Tethys 11: 49-56. Paris.

Prado, J.L.; Alberdi, M.T.; Azanza, B.; Sánchez, B.; Frassinetti, D. 2005. The Pleistocene Gomphotheriidae (Proboscidea) from South America. Quaternary International 126: 21-30.

Tamayo, M.; Frassinetti, D. 1980. Catálogo de los mamíferos fósiles y vivientes de Chile. Boletín del Museo Nacional de Historia Natural 37: 323405. Santiago.

Wyss, A.R.; Norell, M.A.; Flynn, J.J.; Novacek, M.J.; Charrier, R.; McKenna, M.C.; Swisher III, C.C.; Frassinetti, D.; Salinas, P.; Jin, M. 1990. A new early Tertiary mammal fauna, from Central Chile: Implications for Andean stratigraphy and tectonics. Journal of Vertebrate Paleontology 10: 518-522.

\section{New taxa}

\section{Species}

\section{MOLLUSCA}

angoli, Palaeomelon Nielsen \& Frassinetti, 2007a

antiqua, Tegula (Agathistoma) Nielsen, Frassinetti \& Bandel, 2004

antu, Heliacus (Grandeliacus) Nielsen \& Frassinetti, 2007b

austropacifica, Tegula (Chlorostoma) Nielsen, Frassinetti \& Bandel, 2004

bahamondei, Heliacus (Torinista) Frassinetti \& Covacevich, 1981a

bieleri, Solatisonax Nielsen \& Frassinetti, 2007b

biroi, Epitonium Frassinetti \& Covacevich, 1995

caupolicani, Adelomelon Nielsen \& Frassinetti, 2007a

chilena, Tegula (Chlorostoma) Nielsen, Frassinetti \& Bandel, 2004

chilensis, Phos Frassinetti, 2000

chilota, Mulinia Frassinetti, 1997

chonos, Heliacus (Torinista) Nielsen \& Frassinetti, 2007b

colocoloi, Adelomelon Nielsen \& Frassinetti, 2007a

covacevichi, Calliostoma (Otukaia) Nielsen, Frassinetti \& Bandel, 2004

covacevichi, Ipunina Nielsen \& Frassinetti, 2008

covacevichi, Limatula Frassinetti, 1997

covacevichi, Trophon Frassinetti, 2000

crassus, Penion Frassinetti, 2000

crossletensis, Cancellaria Covacevich \& Frassinetti, 1986

diversum, Penion Frassinetti, 2000

fragilis, Diodora Nielsen, Frassinetti \& Bandel, 2004

galvarinoi, Pachycymbiola? Nielsen \& Frassinetti, 2007a

gayana, Ficus Covacevich \& Frassinetti, 1980

guamblinensis, Polinices (Euspira) Frassinetti \& Covacevich, 1995

hero, Xymenopsis? Frassinetti \& Covacevich, 1995

huilliche, Trophon Frassinetti, 2000

ibariformis, Glycymeris (Glycymerita) Frassinetti \& Covacevich, 1984

ignorata, Hindsiclava Frassinetti \& Covacevich, 1995

inti, Architectonica (Intitectonica) Frassinetti \& Covacevich, 1982

kleppi, Calliostoma Nielsen, Frassinetti \& Bandel, 2004

lautaroi, Miomelon Nielsen \& Frassinetti, 2007a

maitenlahuensis, Triumphis Nielsen \& Frassinetti, 2003

mapucherum, Calliostoma Nielsen, Frassinetti \& Bandel, 2004

matanzana, Fagnastesia Nielsen, Frassinetti \& Bandel, 2004 
matanzensis, Tegula (Chlorostoma) Nielsen, Frassinetti \& Bandel, 2004

mcleani, Bathybembix Nielsen, Frassinetti \& Bandel, 2004

miocenica, Diloma Nielsen, Frassinetti \& Bandel, 2004

nassariformis, Cancellaria Covacevich \& Frassinetti, 1986

navidadensis, Amiantis (Amiantis) Frassinetti \& Covacevich, 1993

navidadensis, Architectonica (Discotectonica) Frassinetti \& Covacevich, 1982

parcus, Trophon Frassinetti, 2000

pelantaroi, Miomelon? Nielsen \& Frassinetti, 2007a

pupuyana, Diodora Nielsen, Frassinetti \& Bandel, 2004

reconditus, Adelomelon Frassinetti, 2000

remotum, Cymatium Frassinetti \& Covacevich, 1995

reyesi, Cancellaria Covacevich \& Frassinetti, 1986

riorapelensis, Monilea Nielsen, Frassinetti \& Bandel, 2004

rodulfi, Chione (Chione) Frassinetti \& Covacevich, 1993

rudolphi, Cancellaria Covacevich \& Frassinetti, 1986

senex, Cryptogemma Frassinetti \& Covacevich, 1995

subaraucana, Nucula (Leionucula) Frassinetti, 1997

taitaoensis, Glycymeris (Glycymeris) Frassinetti \& Covacevich, 1993

taverai, Glycymeris Frassinetti \& Covacevich, 1984

taverai, Heliacus (Torinista) Frassinetti \& Covacevich, 1981a

tucapeli, Palaeomelon Nielsen \& Frassinetti, 2007a

vermeiji, Macron Nielsen \& Frassinetti, 2003

vetulus, Trophon Frassinetti, 2000

\section{VERTEBRATA}

smeti, Palaeothenes Flynn, Nov

acek, Dodson, Frassinetti, McKenna, Norell, Sears, Swisher III \& Wyss, 2002

\section{Genera}

\section{MOLLUSCA}

Diconoficus Covacevich \& Frassinetti, 1983

Fagnastesia Nielsen, Frassinetti \& Bandel, 2004

Intitectonica Frassinetti \& Covacevich, 1982

Ipunina Nielsen \& Frassinetti, 2008

Matanziella Frassinetti, 1978

Palaeomelon Nielsen \& Frassinetti, 2007a

\section{CRUSTACEA}

Lebucarcinus Bahamonde \& Frassinetti, 1980 